\title{
Achados audiológicos e queixas relacionadas à audição dos motoristas de ônibus urbano
}

\author{
Audiologic findings and auditory-related complaints \\ of urban bus drivers
}

\author{
Adriana Lacerda ${ }^{1}$, Gisele Figueiredo ${ }^{2}$, Jeane Massarolo Neto ${ }^{3}$, Jair Mendes Marques ${ }^{4}$
}

\begin{abstract}
RESUMO
Objetivo: Investigar os achados audiológicos e as queixas relacionadas à audição dos motoristas de ônibus urbano. Métodos: A amostra foi constituída de 24 motoristas de ônibus da cidade de Francisco Beltrão (PR), com média de idade de 44 anos e três meses e com tempo de exposição total ao ruído de tráfego de 18,8 anos. Aplicou-se uma anamnese com questões referentes à história de saúde geral e auditiva, hábitos de vida e história ocupacional. Posteriormente, realizou-se a audiometria convencional analisada segundo a Portaria 19 do Ministério do Trabalho (1998) e os testes de percepção da fala. Resultados: Com relação aos problemas que acometem a audição, 66,6\% dos motoristas não referem dificuldades para ouvir, 29,2\% referem zumbido e 12,3\% referem ocasionalmente sensação de plenitude auricular. As audiometrias demonstraram que $70 \%$ dos participantes apresentaram audiogramas sugestivos de perda auditiva induzida por ruído. As frequências mais acometidas foram 4000 e $6000 \mathrm{~Hz}$ caracterizando configuração em entalhe bilateral em 83,3\% dos casos. Conclusão: Verifica-se a necessidade do desenvolvimento de ações preventivas voltados à saúde auditiva visando à melhoria das condições de trabalho e a preservação auditiva destes motoristas.
\end{abstract}

Descritores: Efeitos do ruído/diagnóstico; Perda auditiva provocada por ruído; Ruído ocupacional; Ruído dos transportes; Anamnese; Hábitos; Exposição ocupacional; Audiometria; Testes auditivos

\section{INTRODUÇÃO}

A crescente urbanização tem contribuído para o aumento dos níveis de ruído nas cidades, sendo o ruído do tráfego um dos principais responsáveis pela poluição sonora urbana. A exposição ocupacional ao ruído, gerado pelo tráfego de veículos, é um fator preocupante para a saúde auditiva dos motoristas profissionais nos tempos atuais, e vem sendo alvo de inúmeros estudos.

Pesquisas realizadas revelaram que dentre os veículos automotores, o que mais contribui para a poluição sonora urbana são os ônibus, seguida por outros, como as ambulâncias, os

Trabalho realizado na Universidade Tuiuti do Paraná - UTP - Curitiba (PR), Brasil.

(1) Doutora, Professora do Curso de Especialização em Audiologia da Universidade Tuiuti do Paraná - UTP - Curitiba (PR), Brasil.

(2) Especialista em Audiologia; Fonoaudióloga clínica - Francisco Beltrão (PR), Brasil.

(3) Especialista em Audiologia; Fonoaudióloga clínica - Francisco Beltrão (PR), Brasil.

(4) Doutor, Professor do Programa de Mestrado e Doutorado em Distúrbios da Comunicação da Universidade Tuiuti do Paraná - UTP - Curitiba (PR), Brasil. Endereço para correspondência: Adriana Lacerda. R. Sidney Antonio Rangel Santos, 238, Santo Inácio, Curitiba (PR),Brasil, CEP: 82010-330. E-mail: adriana.lacerda@utp.br

Recebido em: 20/2/2009; Aceito em: 21/1/2010 caminhões e as $\operatorname{motos}^{(1)}$. Os autores referiram ainda que a má conservação dos veículos automotores, a falta de isolamento acústico dos motores e escapamentos, o atrito com o asfalto, a má conservação da pavimentação das vias públicas e as buzinas, são fatores que influenciam na produção do ruído urbano ${ }^{(1)}$.

Trabalhos científicos avaliaram os ruídos presentes em ônibus urbanos e revelam que a maioria deles produz níveis de pressão sonora (NPS) acima dos limites de tolerância previstos na norma regulamentadora de número 15 do Ministério do Trabalho (NR-15) ${ }^{(2-6)}$.

Sabe-se que as fontes geradoras de ruídos no interior dos veículos advêm principalmente do motor e que os NPS podem alterar o desempenho das atividades dos motoristas, podendo contribuir para a ocorrência de acidentes envolvendo ônibus, além de causar problemas auditivos nos motoristas ${ }^{(4,6)}$.

Um estudo em Florianópolis (SC) avaliou o ruído de 17 ônibus urbanos e concluiu que dez apresentaram NPS iguais ou superiores a $85 \mathrm{~dB}(\mathrm{NA})$. Dos sete ônibus cujo ruído foi menor que $85 \mathrm{~dB}(\mathrm{~A})$, quatro têm motor traseiro. O maior NPS registrado foi de $95,6 \mathrm{~dB}(\mathrm{~A})^{(4)}$.

Segundo a Organização Mundial de Saúde (OMS), o limite tolerável ao ouvido humano é de $65 \mathrm{~dB}(\mathrm{~A})$, sendo que acima deste nível o organismo pode sofrer estresse, o qual aumenta o risco de doenças. Com ruídos acima de $85 \mathrm{~dB}$ (A), aumenta o risco de comprometimento auditivo, podendo causar efeitos 
auditivos, como a Perda Auditiva Induzida pelo Ruído (PAIR) e/ou efeitos extra-auditivos ${ }^{(7)}$. Sabe-se também que, conforme a susceptibilidade individual, o tempo de exposição e o nível de ruído ao qual a pessoa está exposta, aumentam o risco de sofrer danos à saúde ${ }^{(7)}$.

Estudos realizados com motoristas de ônibus demonstraram a prevalência de PAIR variando entre $32 \%$ e $62,8 \%{ }^{(8-15)}$. A idade e o tempo de serviço exerceram influência sobre a ocorrência desta alteração; constatou-se que quanto maior o tempo de exposição, maior o número de frequências atin$\operatorname{gidas}^{(9,10)}$. Observou-se ainda que houve predominância de audiogramas sugestivos de PAIR para os motoristas com tempo de exposição superior a seis anos ${ }^{(12,13,16)}$. Observa-se maior ocorrência de PAIR em motoristas que trabalham em ônibus com motor dianteiro, produzindo ruído intermitente com variação de intensidade ${ }^{(4,13-15)}$.

Atualmente sabemos que a PAIR pode trazer prejuízos ao indivíduo e pode interferir significativamente em seu desempenho profissional. Portanto, conhecer a saúde auditiva dos motoristas de ônibus urbano constitui o ponto de partida para que se possa propor e desenvolver, juntamente com as empresas de transporte, ações de promoção, prevenção e reabilitação auditiva, que dizem respeito à saúde ocupacional.

Diante do exposto, o objetivo deste estudo foi de investigar os achadosaudiológicos e as queixas relacionadas à audição dos motoristas de ônibus urbano.

\section{MÉTODOS}

O trabalho caracteriza-se como um estudo transversal realizado no Município de Francisco Beltrão, no estado do Paraná, com motoristas funcionários de duas empresas de transporte coletivo urbano, que utiliza veículos do tipo ônibus.

A primeira empresa dispunha de dez funcionários que exerciam a função de motorista e possuía uma frota composta por nove ônibus sendo dois da marca Mercedes ${ }^{\circledR}$, modelo 1418 e sete da marca Volkswagen ${ }^{\circledR}$, modelos: 1621,1622 e 1418. O nível de ruído médio produzido pelos motores da frota, descrito no Programa de Prevenção do Risco Ambiental (PPRA) de 2005, foi de 80,4 dB (A). A segunda empresa dispunha de 14 funcionários que exerciam a função de motorista e possuía uma frota composta por dez ônibus da marca Volkswagen $®$, modelo1621. O nível de ruído médio produzido pelos motores da frota, descrito no PPRA de 2005, foi $81 \mathrm{~dB}$ (A).

Os sujeitos da pesquisa foram 24 motoristas com turno de trabalho de seis horas diárias, não utilizando protetores auriculares ou usufruindo intervalos de repouso durante a jornada de trabalho e não apresentando exposição às atividades extra-ocupacionais ruidosas.

Esta pesquisa foi aceita pelo Comitê de Ética e Pesquisa da Universidade Tuiuti do Paraná (UTP) e aprovada sob número 0001/2007. Os motoristas participantes da pesquisa receberam uma carta de apresentação e foram orientados a assinar um Termo de Consentimento Livre e Esclarecido para viabilizar a utilização dos dados colhidos e garantindo o sigilo a respeito da identidade dos informantes.

Inicialmente, realizou-se uma anamnese a fim de conhecer o histórico sobre a saúde geral e sobre a audição, bem como os hábitos de vida e o histórico ocupacional dos motoristas entrevistados. Posteriormente, os participantes foram submetidos a uma avaliação auditiva composta por testes audiológicos subjetivos. Antecedendo aos exames audiológicos, realizouse a inspeção do meato acústico externo, para verificação da possibilidade ou não da realização dos exames, utilizando-se do meatoscópio modelo TK 007, marca Missouriß .

Os exames audiológicos foram realizados em cabina acústica, seguindo as recomendações internacionais (ISO 8253), utilizando audiômetro da marca Maico®, modelo MA 41, calibrado de acordo com a norma internacional ANSI S.31-1999 e repouso acústico de no mínimo 14 horas, antes da realização do teste, seguindo os critérios da Portaria 19 do Ministério do Trabalho (1998) ${ }^{(16)}$.

Realizou-se audiometria tonal por Via Aérea (VA), nas frequências de $250 \mathrm{~Hz}$ a $8000 \mathrm{~Hz}$, por Via Óssea (VO) para as frequências de $500 \mathrm{~Hz}$ a $4000 \mathrm{~Hz}$ somente quando os limiares auditivos de VA encontravam-se superiores a $25 \mathrm{~dB}$ (NA); testes de percepção da fala, mais especificamente, os testes de Limiar de Reconhecimento de Fala (SRT) e Índice Percentual de Reconhecimento de Fala (IPRF).

As audiometrias foram classificadas conforme o critério da Portaria 19 do Ministério do Trabalho (1998) ${ }^{(16)}$. Segundo esta Portaria, considera-se dentro do limite de normalidade, os casos cujo audiograma apresenta limiares auditivos menores ou iguais a $25 \mathrm{~dB}$ (NA), em todas as frequências examinadas. Considerase sugestivo de PAIR, os casos cujo audiograma, nas frequências de 3000 e/ou 4000 e/ou $6000 \mathrm{~Hz}$ apresentarem limiares auditivos superiores a $25 \mathrm{~dB}(\mathrm{NA})$, tanto no teste VA quanto por VO, em um ou em ambos os lados. São consideradas outras causas, os casos cujo audiograma não se enquadre nas descrições acima.

Os exames audiológicos, assim como a ananmese, foram analisados estatisticamente. As características do grupo e a prevalência da perda auditiva, classificada conforme critério da Portaria 19 do Ministério do Trabalho (1998) ${ }^{(16)}$,foram o foco das análises. Para as análises do teste de diferenças de proporções o nível de significância usado foi de 0,05 (5\%).

\section{RESULTADOS}

A pesquisa envolveu 24 participantes (um do gênero feminino e 23 do gênero masculino), com idade média de 44 anos e três meses (mínima de 28 anos e máxima de 63 anos), exercendo a função de motoristas de ônibus urbano e apresentando uma jornada de trabalho de seis horas diárias.

A média de tempo de trabalho no exercício da função de motorista na empresa atual foi de seis anos e oito meses (desvio padrão de 2,96 anos), distribuídos em períodos de zero a cinco anos $(33,3 \%)$, de seis a dez anos $(58,3 \%)$ e de 11 a 15 anos $(8,3 \%)$. A média de tempo de trabalho no exercício da função de motorista em empresas anteriores foi de oito anos (desvio padrão de 3,82 anos).

A média de tempo de trabalho total exposto ao ruído de tráfego, na empresa atual ou em anteriores, seja como motorista ou como cobrador, foi de 18 anos e oito meses (desvio padrão de 9,57 anos), distribuídos em períodos de 21 a 30 anos (37,5\%), seguidos pelo período de zero a dez anos $(33,3 \%)$ e de 11 a 20 anos $(25 \%)$. 
Tabela 1. Hábitos dos motoristas e problemas relacionados à saúde ou audição

\begin{tabular}{|c|c|c|c|c|}
\hline \multirow[t]{2}{*}{ Hábitos } & Não & \multirow{2}{*}{\multicolumn{2}{|c|}{$\begin{array}{c}\text { Sim } \\
N(\%)\end{array}$}} & \multirow[t]{2}{*}{ Valor de $p$} \\
\hline & $\mathrm{N}(\%)$ & & & \\
\hline Fumante & $14(58,3)$ & $10(41,7)$ & & 0,2561 \\
\hline Uso de bebida alcoólica & $18(75)$ & $6(25)$ & & $0,0012^{*}$ \\
\hline Uso de bebida estimulante & $4(16,7)$ & $20(83,3)$ & & $0,0000^{*}$ \\
\hline Uso regular de medicamento & $24(100)$ & $0(0)$ & & $0,0139^{*}$ \\
\hline \multirow[t]{2}{*}{ Problemas de saúde } & Não & Sim & & \multirow[t]{2}{*}{ Valor de $p$} \\
\hline & $\mathrm{N}(\%)$ & $\mathrm{N}(\%)$ & & \\
\hline Problema de saúde & $18(75)$ & $6(25)$ & & $0,0012^{*}$ \\
\hline Tratamento & $21(87,5)$ & $3(12,5)$ & & $0,0000^{*}$ \\
\hline \multirow[t]{2}{*}{ Problemas auditivos } & Não & Sim & Às vezes & \multirow[t]{2}{*}{ Valor de $p$} \\
\hline & $\mathrm{N}(\%)$ & $\mathrm{N}(\%)$ & $\mathrm{N}(\%)$ & \\
\hline Perda auditiva por explosão & $21(87,5)$ & $3(12,5)$ & - & $0,0000^{*}$ \\
\hline Dificuldade para ouvir & $16(66,6)$ & $1(4,2)$ & $7(29,2)$ & $0,0260^{*}$ \\
\hline Plenitude auricular & $21(87,5)$ & $0(0)$ & $3(12,5)$ & $0,0000^{*}$ \\
\hline Problemas de ouvido & $24(100)$ & $0(0)$ & - & $0,0139^{*}$ \\
\hline Operação de orelha & $24(100)$ & $0(0)$ & - & $0,0139^{*}$ \\
\hline Zumbido & $17(70,8)$ & $4(16,7)$ & $3(12,5)$ & $0,0005^{*}$ \\
\hline
\end{tabular}

* Valores estatisticamente significantes $(p<0,05)$

Nota: Para aplicação do teste de diferença de proporções envolvendo problemas auditivos, as categorias sim e às vezes foram consideradas como uma única categoria.

Na Tabela 1 são apresentados os resultados obtidos quanto aos hábitos de vida dos motoristas com relação ao consumo de cigarro, bebidas alcoólicas ou estimulantes, uso de medicamentos, problemas de saúde e problemas relacionados à audição.

A maior parte dos indivíduos consumia diariamente algum tipo de bebida estimulante $(83,3 \%)$, sendo o café a mais citada (92\%). Pouco mais da metade dos motoristas entrevistados $(58,3 \%)$ eram fumantes, consumindo até uma carteira por dia (Tabela 1).

Observou-se que 75\% dos sujeitos atualmente não referiram qualquer problema de saúde. Os $25 \%$ restantes, relatam que já apresentaram ou apresentam problemas de saúde, tais como: dores de cabeça (40\%), alteração do colesterol (20\%), sinusite (20\%) e hepatite B (20\%). Contudo, $87,5 \%$ não realizaram tratamento adequado.

Com relação aos problemas que acometem a audição, $66,6 \%$ não referiramdificuldades para ouvir, $29,2 \%$ referiram zumbido e $12,3 \%$ referiram ocasionalmente sensação de plenitude auricular.

O teste de diferença de proporções mostrou que, para os hábitos de vida o não uso de bebida alcoólica e de medicamento foi significativamente maior que o uso dos mesmos, enquanto que o uso de bebida estimulante foi significativamente maior que o não uso. Para os problemas de saúde e auditivos, a proporção dos participantes que não apresentam problemas foi significativamente maior.

Considerando-se a queixa de zumbido $(29,2 \%)$, ressaltouse que a média de idade dos participantes para o surgimento do sintoma foi de 40 anos e a configuração dos audiogramas em todos os casos foram os sugestivos de PAIR.

Na Tabela 2 são apresentados os resultados obtidos quanto às características do zumbido apresentado por sete dos motoristas participantes da pesquisa $(29,2 \%)$.

Observou-se que o zumbido se manifesta diariamente em ambas as orelhas, sendo que o período do dia em que mais perturba é o noturno. Verificou-se que $85,8 \%$ dos sujeitos re-
Tabela 2. Características do zumbido

\begin{tabular}{lcc}
\hline Característica do zumbido & Resultado & $\begin{array}{c}\text { Frequência } \\
\mathrm{N}(\%)\end{array}$ \\
\hline Lateralidade & Bilateral & $7(100)$ \\
Tipo & Pitch agudo & $7(100)$ \\
Intensidade & Médio & $6(85,8)$ \\
Frequência & Baixo & $1(14,2)$ \\
& Diariamente & $6(85,8)$ \\
Duração & Semanalmente & $1(14,2)$ \\
& Mais de um minuto & $6(85,8)$ \\
Tempo de zumbido (anos) & O tempo todo & $1(14,2)$ \\
& De um a cinco anos & $2(28,4)$ \\
& De seis a dez anos & $4(57,4)$ \\
Hora que mais se manifesta & Há mais de dez anos & $1(14,2)$ \\
\hline
\end{tabular}

feriram que o tipo do zumbido é semelhante a um pitch agudo, de intensidade média, com manifestação diária e tem duração maior do que um minuto. Ressaltou-se ainda que $57.4 \%$ dos participantes convivem com o zumbido por um período de tempo entre seis a dez anos.

A avaliação audiológica demonstrou que $100 \%$ dos participantes não apresentaram obstruções na inspeção do meato acústico externo para a realização da audiometria. Constatouse na audiometria que 17 motoristas (70\%) apresentaram audiogramas sugestivos de PAIR, quatro apresentaram audição normal (17\%) e três apresentaram outros tipos de perdas auditivas (13\%), classificadas segundo a Portaria 19 do Ministério do Trabalho (1998).

Quanto à lateralidade da PAIR, foi possível observar que em $83,3 \%$ dos casos a perda auditiva era bilateral, e em 16,6 $\%$ dos casos, unilateral com predominância de perda auditiva à direita. As frequências mais acometidas foram 4000 e $6000 \mathrm{~Hz}$, caracterizando configuração audiométrica em entalhe. 
Tabela 3. Estatística descritiva sobre a classificação da audiometria segundo a Portaria 19 do Ministério do Trabalho (1998) em função da idade (anos) e o tempo de trabalho exposto ao ruído de tráfego

\begin{tabular}{|c|c|c|c|c|c|c|c|c|c|}
\hline \multirow{3}{*}{$\begin{array}{l}\text { Classificação } \\
\text { audiométrica }\end{array}$} & \multirow{3}{*}{ N (\%) } & \multirow{2}{*}{\multicolumn{2}{|c|}{ Idade (anos) }} & \multicolumn{6}{|c|}{ Tempo de trabalho (anos) } \\
\hline & & & & \multicolumn{2}{|c|}{$\begin{array}{c}\text { Empresa atual como } \\
\text { motorista }\end{array}$} & \multicolumn{2}{|c|}{$\begin{array}{c}\text { Empresas anteriores como } \\
\text { motorista }\end{array}$} & \multicolumn{2}{|c|}{$\begin{array}{c}\text { Total como motorista ou } \\
\text { cobrador }\end{array}$} \\
\hline & & Média & DP & Média & DP & Média & $\mathrm{DP}$ & Média & $\mathrm{DP}$ \\
\hline Normal & $4(17)$ & 39,75 & 10,87 & 6,25 & 2,06 & - & - & - & - \\
\hline PAIR & $17(70)$ & 46 & 9,27 & 6,64 & 3,18 & 8,20 & 4,23 & 21,61 & 8,64 \\
\hline Outras causas & $3(13)$ & 38 & 1,73 & 6,87 & 4,59 & - & - & 14,33 & 8,08 \\
\hline
\end{tabular}

Legenda: DP = desvio-padrão

Na Tabela 3 são apresentados os resultados da classificação da audiometria segundo a Portaria 19 do Ministério do Trabalho (1998) em função da idade (anos) e do tempo de trabalho exposto ao ruído de tráfego.

Os participantes que apresentaram audiometrias sugestivas de PAIR possuíam média de idade (46 anos) e tempo de trabalho total exposto ao ruído de tráfego como motorista ou cobrador (21,61 anos) superiores aos demais participantes que apresentaram audiometrias classificadas como normais ou outras causas.

Na Figura 1 é possível observar a comparação entre os limiares médios da orelha direita dos motoristas distribuídos segundo a classificação audiométrica sugeridapela Portaria 19 do Ministério do Trabalho (1998).

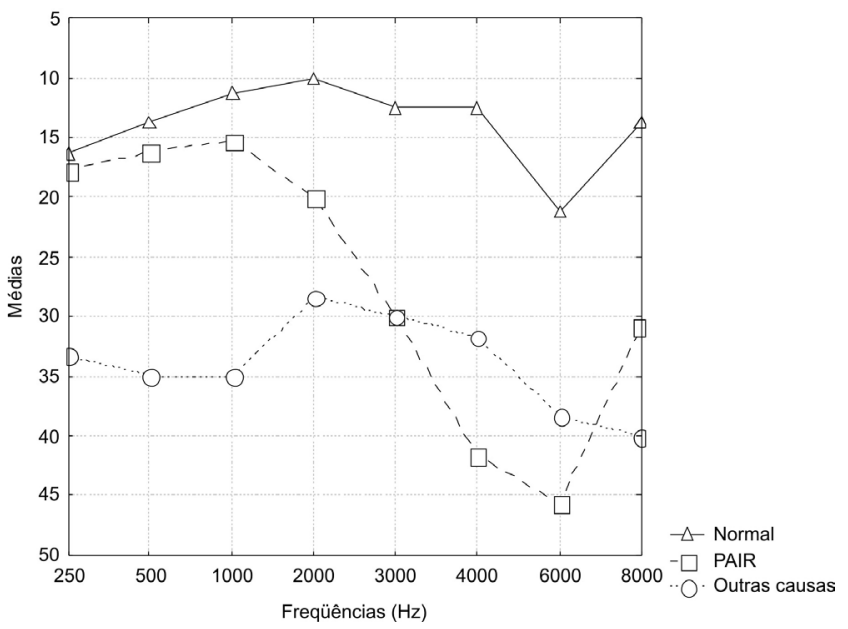

Figura 1. Comparação entre os limiares médios da orelha direita dos motoristas avaliados distribuídos segundo a classificação audiométrica proposta pela Portaria 19 do Ministério do Trabalho, 1998 ${ }^{(16)}$

Na Figura 2 é possível observar a comparação entre os limiares médios da orelha esquerda dos motoristas distribuídos segundo a classificação audiométrica sugeridapela Portaria 19 do Ministério do Trabalho (1998).

O grupo com audiograma normal apresentou configuração de curva audiométrica em entalhe bilateral na frequência de $6000 \mathrm{~Hz}$, assim como o grupo com audiograma sugestivo de PAIR apresentou entalhe audiométrico bilateral em 4000 e $6000 \mathrm{~Hz}$. Já o grupo com audiograma sugestivo de outras causas apresentou configuração de curva audiométrica em "U" invertido para a orelha direita e descendente para a orelha esquerda.

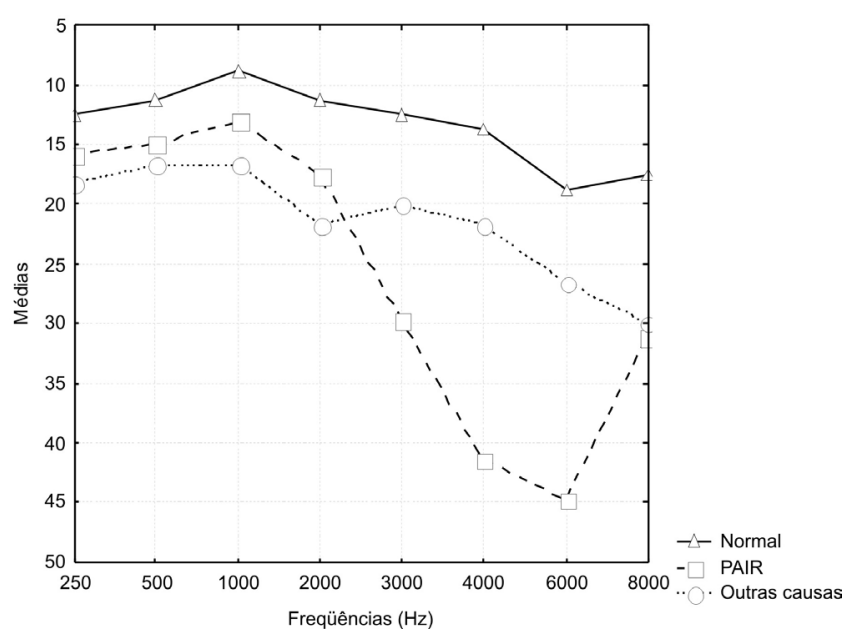

Figura 2. Comparação entre os limiares médios da orelha esquerda dos motoristas avaliados distribuídos segundo classificação audiométrica proposta pela Portaria 19 do Ministério do Trabalho (1998)(16)

Com relação aos testes de percepção de fala, $100 \%$ dos participantes apresentaram normalidade tanto nos testes de Limiar de Reconhecimento de Fala (SRT) quanto no Índice Percentual de Reconhecimento de Fala (IPRF).

$\mathrm{O}$ teste de diferença de proporções entre os grupos de normais e alterados (PAIR + outras causas) não resultou em nenhuma diferença significativa com relação aos hábitos e ou aos problemas de saúde e auditivos $(\mathrm{p}<0,05)$.

\section{DISCUSSÃO}

Este estudo teve por objetivo investigar os achadosaudiológicos e as queixas relacionadas à audição em um grupo composto por 24 motoristas de transporte coletivo urbano.

Nesta população, os resultados relacionados às queixas auditivas demonstraram que $66,6 \%$ dos participantes referiram não apresentar dificuldades para ouvir, 29,2\% apresentaram zumbido e $12,3 \%$ referiram ocasionalmente sensação de plenitude auricular (Tabelas 1 e 2).

É sabido que o zumbido, independentemente da queixa auditiva, é um sintoma auditivo muito relatado por indivíduos expostos ao ruído ${ }^{(11,17-20)}$, sendo a duração da exposição e a severidade do ruído significantemente associados com o sintoma ${ }^{(21)}$. Em portadores de PAIR, os danos às células ciliadas externas poderão dar início ao zumbido como um sintoma de um mau funcionamento do sistema auditivo e 
sua prevalência aumenta conforme a evolução da perda auditiva ${ }^{(22)}$.

Pesquisadores relatam que $50 \%$ dos pacientes com queixa de zumbido melhoram significativamente quando deixam de fumar ou diminuem a ingestão de cafeína. Acredita-se que a cafeína e a nicotina poderiam piorar o quadro de zumbido por serem estimulantes e por provocarem constrição dos vasos sanguíneos ${ }^{(23)}$. Sabe-se ainda, que a idade e o hábito de fumar, associados com a exposição ao ruído, são fatores de risco para a audição e poderiam contribuir para o aparecimento do zumbido e/ou agravamento da PAIR para a população ${ }^{(24-27)}$. Diante do exposto, suspeita-se que o hábito de fumar $(58,3 \%$, até uma carteira por dia) e de consumir diariamente algum tipo de bebida estimulante $(83,3 \%$, sendo o café a mais citada), associados à média de idade dos participantes (44 anos) poderiam contribuir para o surgimento do zumbido e piora da PAIR nesta população (Tabela1).

Com relação aos achados audiológicos, verificou-se que $70 \%$ dos motoristas participantes da pesquisa apresentam audiogramas sugestivos de PAIR (Tabela 3), sendo 83,3\% bilaterale $16,6 \%$ unilateral. Dados semelhantes a este estudo foram obtidos em estudos prévios ${ }^{(8,14)}$. Contudo, outros autores constataram uma prevalência de PAIR inferior a 50\%, contrariamente ao observado no nosso estudo ${ }^{(4,9-12,15)}$.

Nesta população, apesar da idade não ter sido controlada, devido ao número reduzido de participantes, observou-se na Tabela 3 um número maior de casos com PAIR e atuando a mais tempo na profissão. Coerentemente com os nossos achados, autores demonstraram que a idade e o tempo de atuação também podem estar significativamente associados a piora da PAIR ${ }^{(9,10,12-14,16)}$.

Observou-se que $100 \%$ da amostra apresentaram teste de percepção da fala normal. Sabe-se que as pessoas acometidas pela PAIR não costumam queixar-se de surdez, como constatado neste estudo (Tabela 1), porém é comum relatarem dificuldade de inteligibilidade de fala em situações de escuta desfavorável, como ambientes ruidosos ${ }^{(28)}$. Desta forma, sugerimos para esta população testes de fala sensibilizados para a observação dos efeitos do ruído na inteligibilidade de fala.

Neste estudo, apesar da prevalência da PAIR ser elevada (70\%), os resultados do PPRA demonstraram que o ruído médio avaliado encontrava-se abaixo do limite de tolerância proposto pela legislação brasileira, que é de $85 \mathrm{~dB}$ (A). Porém cabe ressaltar que todos os motoristas participantes do estudo trabalhavam com ônibus de motor dianteiro do lado direito e sem proteção auditiva. Fato que poderia justificar a piora, mesmo não sendo observadas diferenças significativas no teste estatístico, nos limiares auditivos da orelha direita (Figura1). Conforme pesquisas anteriormente realizadas $^{(5,13-15)}$, o motor dianteiro direito e o não uso da proteção auditiva contribuem para o agravamento da audição.

Ainda segundo a literatura, a vibração e o monóxido de carbono $(\mathrm{CO})$ produzido pelos motores também seriam fatores de risco para a audição dos motoristas de ônibus. Estudos demonstraram que a exposição ao ruído, mesmo abaixo dos limites de tolerância, associada à vibração e ou ao $\mathrm{CO}$ poderiam agravar ou potencializar a PAIR ${ }^{(15,29,30)}$. Neste estudo, a vibração e o $\mathrm{CO}$ não foram avaliados.

Porém, apesar dos participantes apresentarem fatores de risco para audição ${ }^{(5,13-15,24-27,29,30)}$ (idade, hábito de fumar e de ingerir bebidas estimulantes, características do ônibus, ausência de proteção auditiva, e o efeito combinado do ruído com os agentes otoagressores), não foram observadas diferenças significativas $(\mathrm{p}<0,05)$ envolvendo os hábitos, os problemas de saúde e de audição entre o grupo com audição normal comparativamente aos grupos com audição alterada (sugestivo de PAIR e outras causas) (Tabelas 1 e 3). Suspeita-se que o fato da população avaliada se auto-classificar como saudável (Tabela 1) e o número restrito de participantes $(\mathrm{N}=24)$ tenham limitado ou ocultado o efeito combinado dos fatores de risco para audição dos motoristas participantes da pesquisa. Sugerese que estudos sejam realizados para demonstrar a hipótese de agravamento ou potencialização da PAIR ocasionado pelos fatores de risco acima citados. Recomenda-se a criação de um grupo controle para melhor observação dos resultados.

Incentivamosa existência de ações preventivas visando à melhoria das condições de trabalho dos motoristas de ônibus. Sugerimos ações ambientais e coletivas, como o controle do ruído e de outros agentes otoagressores, a manutenção dos veículos, a utilização de ônibus com motores traseiros, a utilização de protetores auditivos adequados para função e pausas durante a jornada de trabalho visando à redução e o controle da exposição ao ruído produzido pelos motores. Ações estas que vêm a prevenir a PAIR, proporcionando uma melhor qualidade de vida aos motoristas, refletindo, assim, no desempenho e satisfação profissional.

Embora tenhamos uma legislação que contemple os direitos e deveres de empregados e empregadores quanto à exposição ao ruído ocupacional, objetivando a prevenção de riscos à saúde e a promoção da saúde auditiva, ainda é precário o interesse, o conhecimento e o desenvolvimento de ações preventivas de todos os envolvidos nesta categoria profissional.

Concluímos que conhecer a saúde auditiva desta população constituiu-se o ponto de partida para que se possa desenvolver, juntamente com as empresas de transporte da cidade de Francisco Beltrão (PR), ações de promoção, prevenção e reabilitação voltadas à saúde auditiva, visando à melhoria das condições de trabalho e a preservação auditiva destes motoristas.

\section{CONCLUSÃO}

Por meio deste estudo pôde-se concluir que os motoristas de ônibus possuíam queixas relacionadas à audição, sendo as mais frequentes o zumbido e a sensação de plenitude auricular. Considerando-se a queixa de zumbido, ressaltou-se que a média de idade dos participantes para o surgimento do sintoma foi de 40 anos e a configuração dos audiogramas, em todos os casos, foi sugestiva de PAIR.

Quanto aos achados audiológicos dos participantes, observou-se que a maioria apresentou alterações auditivas sugestivas de PAIR, com configuração audiométrica em entalhe bilateral, sendo 4000 e $6000 \mathrm{~Hz}$ as frequências mais acometidas. 


\begin{abstract}
Purpose: To investigate audiologic findings and auditory-related complaints of urban bus drivers. Methods: The sample comprised 24 bus drivers from the city of Francisco Beltrão (PR, Brazil), with average age of 44 years and three months, and total time of exposition to traffic noise of 18.8 years, in average. An anamnesis was carried out, with questions referring to general and hearing health history, lifestyle and occupational history. Subsequently, pure tone audiometry and speech perception tests were performed. The results of the audiometry were analyzed according to the Resolution 19 of the Ministry of Labor (1998). Results: Regarding the problems that affect hearing, $66.6 \%$ of the drivers did not report hearing difficulties; $29.2 \%$ reported tinnitus, and $12.3 \%$ reported occasional sensation of aural fullness. Pure tone audiometry showed that $70 \%$ of the participants had results suggestive of noise induced hearing loss. The most affected frequencies were 4 and $6 \mathrm{kHz}$, characterizing bilateral noise induced notch in $83.3 \%$ of the cases. Conclusion: This study showed a need for the development of preventive actions towards hearing health, in order to promote improvements on work conditions and hearing preservation of bus drivers.
\end{abstract}

Keywords: Noise effects/diagnosis; Hearing loss, Noise-induced; Noise, occupational; Noise, transportation; Medical history taking; Habits; Occupational exposure; Audiometry; Hearing tests

\title{
REFERÊNCIAS
}

1. Freitas RGF, Nakamura HY. Perda auditiva induzida por ruído em motoristas de ônibus com motor dianteiro. Saude Rev. 2004;5(10):13-9.

2. Brasil. Ministério do Trabalho e Emprego. Secretaria de Segurança e Saúde no Trabalho. Norma Regulamentadora(NR)-15. Atividades e operações insalubres. [Internet]. [citado 2008Jun]; Disponível em: http:// www.mte.gov.br/legislacao/normas_regulamentadoras/nr_15.pdf

3. Carvalho MJM. Poluição sonora no transito e no interior dos ônibus de Belo Horizonte. Anais do IV Simpósio Brasileiro de Acústica Veícular(SIBRAV); 1997; São Paulo: Sociedade Brasileira de Acústica; 1997.

4. Didoné JA. Perda auditiva dos motoristas de ônibus por exposição ao ruído: medição, análise e proposta de prevenção [tese]. Florianópolis(SC): Universidade Federal de Santa Catarina; 2004. 173p. [Internet]. [citado 2008 Jun]; Disponível em: http://www.tede.ufsc.br/ teses/PEPS4427.pdf

5. Lacerda A, Ribas A, Mendes J, Andrade P. Noise level and its perception by commuters in urban buses of Curitiba. Can Acoust. 2004;32(4):53-9.

6. Fonseca CJ, Nicótera FN, Gronchi CC, Pastorello NA, Alves LC. Avaliação da exposição ao ruído dos motoristas e cobradores de ônibus de São Paulo.São Paulo: FUNDACENTRO; 1993.

7. Berlung B, Lindvall T, Schwela D, editors. Guidelines for Community Noise. Geneva: World Health Organization; 1999.

8. Patwardhan MS, Kolate MM, More TA. To assess effect of noise on hearing ability of bus drivers by audiometry.Indian J Physiol Pharmacol. 1991;35(1):35-8.

9. Talamini ME. A perda auditiva induzida por ruído em motoristas de ônibus. Tuiuti Ciênc Cul. 1994;2(2):41-56.

10. Cordeiro R, Lima-Filho EC, Nascimento LCR. Associação da perda auditiva induzida pelo ruído como tempo acumulado de trabalho entre motoristas e cobradores. Cad Saúde Pública. 1994;10(2):210-21.

11. Martins AL, Alvarenga KF, Bevilacqua MC, CostaFilho OA. Perda Auditiva em motoristas e cobradores de ônibus. Rev Bras Otorrinolaringol. 2001;67(4):467-73.

12. Corrêa Filho HR, CostaLS, HoehneEL, PérezMAG, NascimentoLCR, MouraE C. Perda auditiva induzida por ruído e hipertensão em condutores de ônibus. Rev Saúde Pública.2002;36(6):693-701.

13. Rossi MM, Ferreira Junior M. O impacto do ruído urbano sobre a audição de operadores de tráfego. Rev Bras Med Trab. 2004;2(2):12632.

14. Fernandes JC, Marinho T, Fernandes VM. Avaliação dos níveis de ruído e perda auditiva em motoristas de ônibus na cidade de São Paulo. XI Simpósio de Engenharia de Produção (SIMPEP); 2004 Nov 08-Nov 10; Bauru: Universidade Estadual Paulista-UNESP; 2004

15. Silva LF, Mendes R. Exposição combinada entre ruído e vibração e seus efeitos sobre a audição de trabalhadores. Rev Saúde Pública. 2005;39(1):9-17.

16. Brasil.Norma Regulamentadora (NR) 7 - Programa de controle médico de saúde ocupacional. Portaria No. 19 de 9 de abril de 1998. Estabelece diretrizes e parâmetros mínimos para avaliação e acompanhamento da audição dos trabalhadores expostos a níveis de pressão sonora elevados. DiárioOficial da União. 1994 Dez 30, p. 21278.

17. Chung DY, Gannon RP, Mason K. Factors affecting the prevalence of tinnitus. Audiology.1984;23(5):441-52.

18. Dias A, Cordeiro R, Correte JE, Gonçalves CGO. Associação entre perda auditiva induzida pelo ruído e zumbidos. Cad SaúdePública.2006;22(1):63-8.

19. Sahley TL, Nodar RH. Tinnitus: present and future. CurrOpinOtolaryngol Head Neck Surg. 2001;9(5):323-8.

20. Steinmetz LG, Zelgelboim BS, Lacerda AB, Morata TC, Marques JM. Evaluating tinnitus in industrial hearing loss prevention programs.Int Tinnitus J.2008;14(2):152-8.

21. Sindhusake D, Golding M, Newall P, Rubin G, Jakobsen K, Mitchell P.Risk factors for tinnitus in a population of older adults: the blue mountains hearing study. Ear Hear.2003;24(6):501-7.

22. Olsen S. O Zumbido: resultado da exposição a níveis sonoros excessivos.In: Nudelmann AA, Costa EAC, Seligman J, Ibanez RN. PAIR: Perda auditiva induzida pelo ruído. Vol. 2. Rio de Janeiro(RJ): Revinter; 2001. p. 93-105. Capítulo 7.

23. Schleuning, A. Medical aspects of tinnitus. In: Vernon J, editor. Tinnitus treatment and relief. Boston: Ally and Bacon; 1998.

24. Ferrite S, Santana V. Joint effects of smoking, noise exposure and age on hearing loss. Occup Med (Lond).2005;55(1):48-53.

25. Phaneuf R, Hétu R. An epidemiological perspective of the causes of hearing loss among industrial workers.J Otolaryngol.1990;19(1):31-40.

26. Lim DP, Stephens SD. Clinical investigation of hearing loss in the elderly.ClinOtolaryngol Allied Sci.1991;16(3):288-93.

27. Fransen E, Topsakal V, Hendrickx JJ, Van Laer L, Huyghe JR, VanEyken E, et. al. Occupational noise, smoking, and a high body mass index are risk factors for age-related hearing impairment and moderate alcohol consumption is protective: aEuropean population-based multicenter study. J Assoc Res Otolaryngol.2008;9(3):264-76.

28. DaCosta EA. Brazilian Portuguese speech material and its application in occupational audiology. Audiology. 2001;40(3):123-32.

29. Fernades M, Morata TC. Estudo dos efeitos auditivos e extra-auditivos da exposição ocupacional a ruído e vibração. Rev Bras Otorrinolaringol. 2002;68(5):705-13.

30. Lacerda A, Leroux T, Morata T. Efeitos ototóxicos da exposição ao monóxido de carbono: uma revisão. Pró-fono. 2005;17(3):403-12. 